\title{
Effect of Imazethapyr as Early Post-Emergence Herbicide on Weed Dynamics and Yield of Greengram (Vigna radiata L.)
}

\author{
Girdhari Lal ${ }^{1}$, S.M. Hiremath ${ }^{2}$ and Bheiru Singh ${ }^{3 *}$ \\ ${ }^{1}$ Department of Agronomy, Institute of Agricultural Sciences, Banaras Hindu University, \\ Varanasi-221005, India \\ ${ }^{2}$ Department of Agronomy, ARS, Gadag, UAS, Dharwad-580 005 (Karnatka), India \\ ${ }^{3}$ Krishi Vigyan Kendra, Hanumangarh-II 335523 (Rajasthan), India \\ *Corresponding author
}

\begin{abstract}
A B S T R A C T
\section{Keywords}

Imazethapyr, Early postemergence, Weed control efficiency, Greengram, Weed dynamics

Article Info

\section{Accepted:}

18 September 2018 Available Online: 10 October 2018

A field experiment was conducted during kharif of 2014-15 at Main Agricultural Research Station, Dharwad to study the effect of early post-emergence herbicide Imazethapyr on weed dynamics and yield of greengram. Results revealed that weed free check $\left(\mathrm{T}_{10}\right)$ recorded significantly lower total weed population, total weed dry weight of weeds, higher weed control efficiency (WCE), higher grain yield (833 kg ha-1), haulm yield (2685 kg $\mathrm{ha}^{-1}$ ), number of pods plant ${ }^{-1}(15.49)$, grain weight plant $^{-1}(4.32 \mathrm{~g})$ and gross return (Rs 40,707 ha ${ }^{-1}$ ), However, treatments $\mathrm{T}_{8}$ (Standard check $2 \mathrm{IC}+2 \mathrm{HW}$ at 20 and $40 \mathrm{DAS}$ ) and

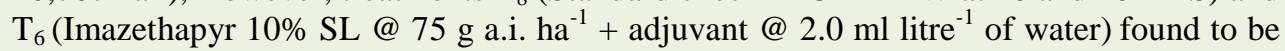
at par with $\mathrm{T}_{10}$. Among the herbicide treatments, $\mathrm{T}_{6}$ recorded significantly higher grain yield $\left(783 \mathrm{~kg} \mathrm{ha}^{-1}\right)$, haulm yield $\left(2528 \mathrm{~kg} \mathrm{ha}^{-1}\right)$, number of pods plant ${ }^{-1}(15.06)$, grain weight plant ${ }^{-1}(4.08 \mathrm{~g})$, gross return (Rs 38,269 ha-1), net return (Rs 19,090 ha-1) and B: C ratio (2.00). It also recorded lower total number of grassy weeds, broad leaved weeds, sedge, total weed dry weight and higher WCE at $40(86.49 \%), 60$ DAS $(82.93 \%)$ and at harvest $(82.30 \%)$.
\end{abstract}

\section{Introduction}

Greengram (Vigna radiata L.) is the third most important pulse crop of India, after chickpea and pigeonpea. It contains about 24.5 per cent protein. The protein is comparatively rich in lysine which is deficient in cereal grains. In India it covers an area of 3.55 million hectares with total production of 1.61 million tonnes and an average productivity of $619 \mathrm{~kg} \mathrm{ha}^{-1}$ (Anon., 2013). The low yields of greengram in India as compared to the world productivity is growing of pulses under marginal and less fertile soil with low inputs and without weed, pest and disease management. Among several factors responsible for lower productivity and grain yield of greengram, weed infestation is one of the major factors. The crop mainly cultivated during kharif season and is infested with various grassy weeds, sedge and broad leaved weeds which emerge simultaneously with the crop plants and rob essential nutrients, space, moisture and sunlight causing substantial loss 
in yield. The weeds, if not controlled during critical period of crop-weed competition, there may be reduction in the yield of greengram depending upon type of weeds and weed intensity. The critical period of crop-weed competition is 45 days (Singh et al., 1996). To avoid the competition during early growth stages, greengram field should kept free from weeds for the first 30-45 days after sowing, after that the crop able to cover the land and takes care of late emerging weeds.

Weed control through hand weeding is tedious, time and labour consuming. So, there is need to evaluate the new post emergence herbicide for weed control in greengram. The herbicide presently available for greengram are narrow spectrum and control only one type of weeds either grassy or broad leaved weeds. Imazethapyr is a broad spectrum herbicide used as early post-emergence in pulses, oilseeds and leguminous crops. The information on use of imazethapyr as a early post-emergence is meager \& hence study was carried out.

\section{Materials and Methods}

A field experiment was undertaken at Main Agricultural Research Station, University of Agricultural Sciences, Dharwad (Karnataka) during kharif season of 2014-15. The experiment was laid out in a randomized complete block design (RCBD) with three replications and ten treatments comprising, Imazethapyr 10\% SL @ 50 g a.i. ha ${ }^{-1}\left(\mathrm{~T}_{1}\right)$, Imazethapyr 10\% SL @ $62.5 \mathrm{~g}$ a.i. ha ${ }^{-1}\left(\mathrm{~T}_{2}\right)$, Imazethapyr 10\% SL @ 75 g a.i. ha ${ }^{-1}\left(\mathrm{~T}_{3}\right)$, Imazethapyr 10\% SL @50 g a.i. ha ${ }^{-1}+$ adjuvant@2.0 ml litre ${ }^{-1}$ of water $\left(\mathrm{T}_{4}\right)$, Imazethapyr 10\% SL @62.5 g a.i. ha ${ }^{-1}+$ adjuvant @2.0 ml litre ${ }^{-1}$ of water $\left(\mathrm{T}_{5}\right)$, Imazethapyr 10\% SL @75 g a.i. ha ${ }^{-1}+$ adjuvant@2.0 ml litre ${ }^{-1}$ of water $\left(\mathrm{T}_{6}\right)$, Quizalofop-ethyl 5\% EC @ $1000 \mathrm{ml}$ litre $^{-1}$ of water $\left(\mathrm{T}_{7}\right)$, standard check (2 Inter-cultivation
+ 2 Hand Weeding at 20 and $40 \mathrm{DAS})\left(\mathrm{T}_{8}\right)$, weedy check $\left(\mathrm{T}_{9}\right)$ and weed free check $\left(\mathrm{T}_{10}\right)$. The experimental soil was black clayey soil (Vertisol) with the $\mathrm{pH} 7.4$, organic carbon $(8 \mathrm{~g}$ $\left.\mathrm{kg}^{-1}\right)$, available $\mathrm{N}\left(248 \mathrm{~kg} \mathrm{ha}^{-1}\right)$, available $\mathrm{P}_{2} \mathrm{O}_{5}$ $\left(25.55 \mathrm{~kg} \mathrm{ha}^{-1}\right)$ and available $\mathrm{K}_{2} \mathrm{O}(396 \mathrm{~kg}$ $\left.\mathrm{ha}^{-1}\right)$. Greengram variety 'Nirmal Gold (NVL1)' was sown on $14^{\text {th }}$ July 2014 at $30 \times 10 \mathrm{~cm}$ spacing using seed rate of $12 \mathrm{~kg} \mathrm{ha}^{-1}$. The recommended fertilizers i.e. $25: 50 \mathrm{~kg} \mathrm{ha}^{-1} \mathrm{~N}$ and $\mathrm{P}_{2} \mathrm{O}_{5}$ were applied at the time of sowing. The total rainfall received during crop growth period was $604.2 \mathrm{~mm}$ and was well distributed. Spraying of chloropyriphos@2 $\mathrm{ml}$ litre $^{-1}$ and bavistin @2 $\mathrm{g} \mathrm{litre}^{-1}$ of water was done for control of sucking insects, pod borers and disease. The early post-emergence herbicide Imazethapyr was sprayed at 23 DAS and other herbicide such as Quizalofop-ethyl was sprayed at the same time. The herbicides were sprayed with knapsack sprayer using 750 litres of spray solution per hectare. Weed population and weed dry weight $\mathrm{m}^{-2}$ were recorded at 20, 40, 60 DAS and at harvest based on which WCE was calculated. The quadrate of 0.25 square meter was used to count the weeds in each plot. The data collected on weeds were transformed through the square root transformation $\sqrt{X}+0.5$ for statistical analysis. The various yield parameters such as number of pods plant ${ }^{-1}$, grain weight plant $^{-1}$, grain yield and haulm yield were recorded at harvest stage of greengram.

\section{Results and Discussion}

The important grassy weeds were Cynodon dactylon and Dinebra retroflexa, and while among the broad-leaved weeds Ageratum conyzoides, Corchorus trilocularis, Digera arvensis and Parthenium hysterophorus were found and among sedge Cyperus rotundus was found in the experimental area. Among the different weed Cyperus rotundus and Cynodon dactylon were dominant. 
Table.1 Total number of grassy weeds, broad leaved weeds and sedge $\left(\mathrm{m}^{-2}\right)$ at different growth stages in soybean as influenced by application of early post-emergence herbicides

\begin{tabular}{|c|c|c|c|c|c|c|c|c|c|c|c|c|}
\hline \multirow[t]{2}{*}{ Treatment } & \multicolumn{3}{|c|}{ Total number of weeds $\left(\mathrm{m}^{-2}\right)$} & \multicolumn{3}{|c|}{$\begin{array}{l}\text { Total number of grassy weeds } \\
\left(\mathrm{m}^{-2}\right)\end{array}$} & \multicolumn{3}{|c|}{$\begin{array}{c}\text { Total number of broad leaved } \\
\text { weeds }\left(\mathrm{m}^{-2}\right)\end{array}$} & \multicolumn{3}{|c|}{ Total number of sedge $\left(\mathrm{m}^{-2}\right)$} \\
\hline & 20 DAS & 40 DAS & 60 DAS & 20 DAS & 40 DAS & 60 DAS & $\begin{array}{c}20 \\
\text { DAS }\end{array}$ & 40 DAS & 60 DAS & $\begin{array}{c}20 \\
\text { DAS }\end{array}$ & $\begin{array}{c}40 \\
\text { DAS }\end{array}$ & $\begin{array}{c}\text { 60 } \\
\text { DAS }\end{array}$ \\
\hline $\mathrm{T}_{1}$ - Imazethapyr 10\% SL $50 \mathrm{~g} \mathrm{ail} \mathrm{ha}^{-1}$ (EPOE) & $\begin{array}{c}11.94 * \\
(144.00)\end{array}$ & $\begin{array}{c}10.05^{*} \\
(101.00)\end{array}$ & $\begin{array}{c}12.45^{*} \\
(154.67)\end{array}$ & $\begin{array}{l}5.14^{*} \\
(26.00)\end{array}$ & $\begin{array}{l}4.91^{*} \\
(23.67)\end{array}$ & $\begin{array}{l}5.74 * \\
(32.67)\end{array}$ & $\begin{array}{l}5.74^{*} \\
(32.67)\end{array}$ & $\begin{array}{c}7.24^{*} \\
(52.00)\end{array}$ & $\begin{array}{l}9.16^{*} \\
(84.00)\end{array}$ & $\begin{array}{l}9.12^{*} \\
(85.33)\end{array}$ & $\begin{array}{l}5.97^{*} \\
(35.33)\end{array}$ & $\begin{array}{l}6.19^{*} \\
(38.00)\end{array}$ \\
\hline $\mathrm{T}_{2}$ - Imazethapyr 10\% SL $62.5 \mathrm{~g}$ a.i ha ${ }^{-1}$ (EPOE) & $\begin{array}{c}11.65 \\
(135.33)\end{array}$ & $\begin{array}{c}9.99 \\
(100.00)\end{array}$ & $\begin{array}{c}11.96 \\
(142.67)\end{array}$ & $\begin{array}{c}4.92 \\
(24.00)\end{array}$ & $\begin{array}{c}4.71 \\
(22.00)\end{array}$ & $\begin{array}{c}5.37 \\
(28.67)\end{array}$ & $\begin{array}{c}6.40 \\
(40.67)\end{array}$ & $\begin{array}{c}7.64 \\
(58.00)\end{array}$ & $\begin{array}{c}8.93 \\
(79.33)\end{array}$ & $\begin{array}{c}8.43 \\
(70.67)\end{array}$ & $\begin{array}{c}5.33 \\
(28.00)\end{array}$ & $\begin{array}{c}5.92 \\
(34.67)\end{array}$ \\
\hline $\mathrm{T}_{3}$ - Imazethapyr 10\% SL $75 \mathrm{~g}$ a.i ha ${ }^{-1}$ (EPOE) & $\begin{array}{c}11.63 \\
(136.00)\end{array}$ & $\begin{array}{c}8.68 \\
(75.33)\end{array}$ & $\begin{array}{c}10.82 \\
(116.67)\end{array}$ & $\begin{array}{c}4.29 \\
(18.00)\end{array}$ & $\begin{array}{c}4.58 \\
(20.67)\end{array}$ & $\begin{array}{c}5.14 \\
(26.00)\end{array}$ & $\begin{array}{c}7.01 \\
(48.67)\end{array}$ & $\begin{array}{c}6.15 \\
(37.33)\end{array}$ & $\begin{array}{c}8.10 \\
(65.33)\end{array}$ & $\begin{array}{c}8.27 \\
(69.33)\end{array}$ & $\begin{array}{c}4.78 \\
(22.67)\end{array}$ & $\begin{array}{c}5.05 \\
(25.33)\end{array}$ \\
\hline $\begin{array}{l}\mathrm{T}_{4}-\text { Imazethapyr } 10 \% \text { SL } 50 \mathrm{~g} \text { a.i ha }{ }^{-1}+\text { Adjuvant @ } 2.0 \mathrm{ml} \mathrm{litre}^{-1} \text { of } \\
\text { water (EPOE) }\end{array}$ & $\begin{array}{c}10.30 \\
(106.67)\end{array}$ & $\begin{array}{c}9.39 \\
(88.67)\end{array}$ & $\begin{array}{c}10.96 \\
(120.00)\end{array}$ & $\begin{array}{c}4.52 \\
(20.00)\end{array}$ & $\begin{array}{c}4.88 \\
(23.33)\end{array}$ & $\begin{array}{c}5.58 \\
(30.67)\end{array}$ & $\begin{array}{c}5.90 \\
(34.67)\end{array}$ & $\begin{array}{c}6.75 \\
(45.33)\end{array}$ & $\begin{array}{c}7.82 \\
(61.67)\end{array}$ & $\begin{array}{c}7.12 \\
(52.00)\end{array}$ & $\begin{array}{c}5.02 \\
(25.33)\end{array}$ & $\begin{array}{c}5.35 \\
(28.67)\end{array}$ \\
\hline $\begin{array}{l}\mathrm{T}_{5} \text {-Imazethapyr 10\% SL 62.5g a.i ha }{ }^{-1}+\text { Adjuvant @ } 2.0 \mathrm{ml} \mathrm{litre}{ }^{-1} \text { of } \\
\text { water (EPOE) }\end{array}$ & $\begin{array}{c}11.48 \\
(131.33)\end{array}$ & $\begin{array}{c}8.67 \\
(75.33)\end{array}$ & $\begin{array}{c}10.38 \\
(107.33)\end{array}$ & $\begin{array}{c}5.03 \\
(25.33)\end{array}$ & $\begin{array}{c}4.67 \\
(21.33)\end{array}$ & $\begin{array}{l}5.05 \\
(25.33)\end{array}$ & $\begin{array}{c}6.62 \\
(43.33)\end{array}$ & $\begin{array}{c}6.25 \\
(38.67)\end{array}$ & $\begin{array}{c}7.37 \\
(54.00)\end{array}$ & $\begin{array}{c}7.90 \\
(62.67)\end{array}$ & $\begin{array}{c}4.46 \\
(20.00)\end{array}$ & $\begin{array}{c}5.30 \\
(28.00)\end{array}$ \\
\hline 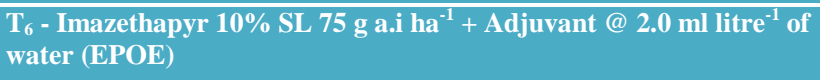 & $\begin{array}{c}10.66 \\
(114.00)\end{array}$ & $\begin{array}{c}6.46 \\
(41.33)\end{array}$ & $\begin{array}{c}8.03 \\
(64.00)\end{array}$ & $\begin{array}{c}4.36 \\
(18.67)\end{array}$ & $\begin{array}{c}3.81 \\
(14.00)\end{array}$ & $\begin{array}{c}4.14 \\
(16.67)\end{array}$ & $\begin{array}{c}5.76 \\
(32.67)\end{array}$ & $\begin{array}{c}4.74 \\
(22.00)\end{array}$ & $\begin{array}{c}5.91 \\
(34.67)\end{array}$ & $\begin{array}{c}7.87 \\
(62.67)\end{array}$ & $\begin{array}{c}2.90 \\
(8.00)\end{array}$ & $\begin{array}{c}3.61 \\
(12.67)\end{array}$ \\
\hline $\mathrm{T}_{8}-$ Standard check (2 IC + $2 \mathrm{HW}$ at 20 and $\left.40 \mathrm{DAS}\right)$ & $\begin{array}{c}11.55 \\
(134.00)\end{array}$ & $\begin{array}{c}5.21 \\
(26.67)\end{array}$ & $\begin{array}{c}6.74 \\
(45.00)\end{array}$ & $\begin{array}{c}4.81 \\
(22.67)\end{array}$ & $\begin{array}{c}3.12 \\
(9.33)\end{array}$ & $\begin{array}{c}3.53 \\
(12.00)\end{array}$ & $\begin{array}{c}5.17 \\
(26.67)\end{array}$ & $\begin{array}{c}3.80 \\
(14.00)\end{array}$ & $\begin{array}{c}4.75 \\
(22.33)\end{array}$ & $\begin{array}{c}9.12 \\
(84.67)\end{array}$ & $\begin{array}{c}2.21 \\
(4.67)\end{array}$ & $\begin{array}{c}3.32 \\
(10.67)\end{array}$ \\
\hline$T_{9}$ - Weedy check & $\begin{array}{c}11.33 \\
(130.00)\end{array}$ & $\begin{array}{c}17.49 \\
(306.00)\end{array}$ & $\begin{array}{c}19.45 \\
(380.00)\end{array}$ & $\begin{array}{c}4.66 \\
(22.00)\end{array}$ & $\begin{array}{c}10.09 \\
(101.33)\end{array}$ & $\begin{array}{c}11.09 \\
(122.67)\end{array}$ & $\begin{array}{c}6.03 \\
(36.00)\end{array}$ & $\begin{array}{c}12.20 \\
(148.67)\end{array}$ & $\begin{array}{c}13.22 \\
(174.67)\end{array}$ & $\begin{array}{c}8.37 \\
(72.00)\end{array}$ & $\begin{array}{c}8.72 \\
(76.00)\end{array}$ & $\begin{array}{c}8.97 \\
(82.67)\end{array}$ \\
\hline$T_{10}$ - Weed free & $\begin{array}{c}3.44 \\
(11.33)\end{array}$ & $\begin{array}{c}2.76 \\
(7.33)\end{array}$ & $\begin{array}{c}3.00 \\
(8.67)\end{array}$ & $\begin{array}{c}1.76 \\
(2.67)\end{array}$ & $\begin{array}{c}1.47 \\
(2.00)\end{array}$ & $\begin{array}{c}1.65 \\
(2.67)\end{array}$ & $\begin{array}{c}2.90 \\
(8.00)\end{array}$ & $\begin{array}{c}2.08 \\
(4.00)\end{array}$ & $\begin{array}{c}2.23 \\
(4.67)\end{array}$ & $\begin{array}{c}1.00 \\
(0.67)\end{array}$ & $\begin{array}{c}1.29 \\
(1.33)\end{array}$ & $\begin{array}{c}1.29 \\
(1.33)\end{array}$ \\
\hline S.Em \pm & 0.44 & 0.27 & 0.36 & 0.34 & 0.19 & 0.25 & 0.28 & 0.24 & 0.31 & 0.50 & 0.25 & 0.32 \\
\hline CD (5\%) & 1.31 & 0.81 & 1.08 & 1.01 & 0.57 & 0.74 & 0.85 & 0.74 & 0.94 & 1.49 & 0.74 & 0.96 \\
\hline
\end{tabular}

Note: EPOE- Early post-emergence (23 DAS), IC - Inter-cultivation (20 \& 40 DAS), HW- Hand weeding (20 \&40 DAS), DAS- Days after sowing

* Transformed values $[\sqrt{ }(\mathrm{x}+0.5)]$, Figures in the parenthesis indicate original values. 
Table.2 Total weed dry weight $\left(\mathrm{g} \mathrm{m}^{-2}\right)$ and weed control efficiency $(\%)$ at different growth stages in greengram and weed index $(\%)$ as influenced by application of early post-emergence herbicides

\begin{tabular}{|c|c|c|c|c|c|c|c|c|}
\hline \multirow[t]{2}{*}{ Treatment } & \multicolumn{4}{|c|}{ Total weed dry weight $\left(\mathrm{g} \mathrm{m}^{-2}\right)$} & \multicolumn{3}{|c|}{ WCE $(\%)$} & \multirow{2}{*}{$\begin{array}{l}\text { Weed } \\
\text { index } \\
(\%)\end{array}$} \\
\hline & 20 DAS & 40 DAS & 60 DAS & $\begin{array}{c}\text { At } \\
\text { harvest }\end{array}$ & $\begin{array}{c}40 \\
\text { DAS }\end{array}$ & $\begin{array}{c}60 \\
\text { DAS }\end{array}$ & $\begin{array}{c}\text { At } \\
\text { harvest }\end{array}$ & \\
\hline $\mathrm{T}_{1^{-}}$Imazethapyr 10\% SL $50 \mathrm{~g}$ a.i ha ${ }^{-1}$ (EPOE) & $\begin{array}{c}4.56^{*} \\
(20.57)\end{array}$ & $\begin{array}{c}5.83^{*} \\
(33.67)\end{array}$ & $\begin{array}{c}7.88^{*} \\
(61.73)\end{array}$ & $\begin{array}{c}7.92^{*} \\
(62.35)\end{array}$ & 67.18 & 58.52 & 58.02 & 29.78 \\
\hline $\mathrm{T}_{2}$ - Imazethapyr 10\% SL $62.5 \mathrm{~g}$ a.i ha ${ }^{-1}$ (EPOE) & $\begin{array}{c}4.45 \\
(19.33)\end{array}$ & $\begin{array}{c}5.80 \\
(33.33)\end{array}$ & $\begin{array}{c}7.56 \\
(56.80)\end{array}$ & $\begin{array}{c}7.60 \\
(57.37)\end{array}$ & 67.54 & 61.87 & 61.17 & 29.49 \\
\hline $\mathrm{T}_{3}$ - Imazethapyr 10\% SL $75 \mathrm{~g}$ a.i ha ${ }^{-1}$ (EPOE) & $\begin{array}{c}4.45 \\
(19.43)\end{array}$ & $\begin{array}{c}5.05 \\
(25.11)\end{array}$ & $\begin{array}{c}6.85 \\
(46.53)\end{array}$ & $\begin{array}{c}6.88 \\
(47.00)\end{array}$ & 75.50 & 68.56 & 68.34 & 23.94 \\
\hline $\begin{array}{l}\mathrm{T}_{4} \text { - Imazethapyr 10\% SL } 50 \mathrm{~g} \text { a.i ha }{ }^{-1}+\text { Adjuvant @ } 2.0 \mathrm{ml} \mathrm{litre}^{-1} \text { of } \\
\text { water (EPOE) }\end{array}$ & $\begin{array}{c}3.95 \\
(15.24)\end{array}$ & $\begin{array}{c}5.45 \\
(29.56)\end{array}$ & $\begin{array}{c}6.96 \\
(48.00)\end{array}$ & $\begin{array}{c}6.99 \\
(48.48)\end{array}$ & 71.28 & 68.16 & 68.10 & 25.91 \\
\hline $\begin{array}{l}\mathrm{T}_{5} \text {-Imazethapyr } 10 \% \text { SL 62.5g a.i ha }{ }^{-1}+\text { Adjuvant @ } 2.0 \mathrm{ml} \mathrm{litre}^{-1} \text { of } \\
\text { water (EPOE) }\end{array}$ & $\begin{array}{c}4.39 \\
(18.76)\end{array}$ & $\begin{array}{c}5.04 \\
(25.11)\end{array}$ & $\begin{array}{c}6.63 \\
(43.47)\end{array}$ & $\begin{array}{c}6.66 \\
(43.90)\end{array}$ & 75.55 & 71.42 & 71.10 & 20.07 \\
\hline $\begin{array}{l}\mathrm{T}_{6} \text { - Imazethapyr 10\% SL } 75 \mathrm{~g} \text { a.i ha }{ }^{-1}+\text { Adjuvant @ } 2.0 \mathrm{ml} \mathrm{litre}{ }^{-1} \text { of } \\
\text { water (EPOE) }\end{array}$ & $\begin{array}{c}4.08 \\
(16.29)\end{array}$ & $\begin{array}{c}3.77 \\
(13.78)\end{array}$ & $\begin{array}{c}5.05 \\
(25.07)\end{array}$ & $\begin{array}{c}5.08 \\
(25.32)\end{array}$ & 86.49 & 82.93 & 82.30 & 6.00 \\
\hline $\mathrm{T}_{7}$ - Quizalofop-ethyl 5\% EC @ $1000 \mathrm{ml} \mathrm{ha}^{-1}$ (EPOE) & $\begin{array}{c}4.31 \\
(18.10)\end{array}$ & $\begin{array}{c}6.31 \\
(39.33)\end{array}$ & $\begin{array}{c}7.55 \\
(56.67)\end{array}$ & $\begin{array}{c}7.59 \\
(57.23)\end{array}$ & 61.32 & 62.17 & 62.06 & 28.46 \\
\hline $\mathrm{T}_{8}-$ Standard check (2 IC + $2 \mathrm{HW}$ at 20 and 40 DAS) & $\begin{array}{c}4.41 \\
(19.14)\end{array}$ & $\begin{array}{c}3.06 \\
(8.89)\end{array}$ & $\begin{array}{c}4.36 \\
(18.53)\end{array}$ & $\begin{array}{c}4.38 \\
(18.72)\end{array}$ & 91.28 & 87.76 & 87.44 & 2.64 \\
\hline $\mathrm{T}_{9}$ - Weedy check & $\begin{array}{c}4.33 \\
(18.57)\end{array}$ & $\begin{array}{c}10.11 \\
(102.00)\end{array}$ & $\begin{array}{c}12.31 \\
(151.87)\end{array}$ & $\begin{array}{c}12.37 \\
(153.39)\end{array}$ & - & - & - & 41.79 \\
\hline$T_{10}-$ Weed free & $\begin{array}{l}1.45 \\
(1.62)\end{array}$ & $\begin{array}{l}1.70 \\
(2.44)\end{array}$ & $\begin{array}{c}1.95 \\
(3.33)\end{array}$ & $\begin{array}{c}2.03 \\
(3.63)\end{array}$ & 97.59 & 97.74 & 97.58 & - \\
\hline S.Em \pm & 0.16 & 0.15 & 0.26 & 0.27 & 1.39 & 1.84 & 2.90 & 4.61 \\
\hline CD (5\%) & 0.49 & 0.46 & 0.79 & 0.82 & 4.17 & 5.52 & 8.70 & 13.69 \\
\hline
\end{tabular}

Note: EPOE- Early post-emergence (23 DAS), IC - Inter-cultivation (20 \& 40 DAS), HW- Hand weeding (20 \&40 DAS), DAS- Days after sowing

* Transformed values $[\sqrt{ }(x+0.5)]$, Figures in the parenthesis indicate original values. 
Table.3 Yield, yield attributes and economics of greengram as influenced by application of early post emergence herbicide

\begin{tabular}{|c|c|c|c|c|c|c|c|c|}
\hline \multirow[t]{2}{*}{ Treatment } & \multicolumn{8}{|c|}{ At harvest } \\
\hline & $\begin{array}{l}\text { Number of } \\
\text { pods plant }^{-1}\end{array}$ & $\begin{array}{c}\text { Grain } \\
\text { weight } \\
\left(\text { g plant }^{-1}\right)\end{array}$ & $\begin{array}{c}\text { Test } \\
\text { weight } \\
\text { (g/ 100( } \\
\text { grains) }\end{array}$ & $\begin{array}{c}\text { Grain } \\
\text { yield } \\
\left(\mathrm{kg} \mathrm{ha}^{-1}\right)\end{array}$ & $\begin{array}{c}\text { Haulm } \\
\text { yield } \\
\left(\mathrm{kg} \mathrm{ha}^{-1}\right)\end{array}$ & $\begin{array}{c}\text { Harvest } \\
\text { index } \\
(\%)\end{array}$ & $\begin{array}{l}\text { Net } \\
\text { return } \\
\left(\text { Rs ha }^{-1}\right)\end{array}$ & $\begin{array}{l}\text { B:C } \\
\text { Ratio }\end{array}$ \\
\hline $\mathrm{T}_{1}$ - Imazethapyr 10\% SL $50 \mathrm{~g}$ a.i ha ${ }^{-1}$ (EPOE) & 10.51 & 2.94 & 48.82 & 586 & 2065 & 22.06 & 10604 & 1.58 \\
\hline $\mathrm{T}_{2}$ - Imazethapyr 10\% SL $62.5 \mathrm{~g} \mathrm{a} . \mathrm{i} \mathrm{ha}{ }^{-1}$ (EPOE) & 10.66 & 2.98 & 48.96 & 588 & 2070 & 22.16 & 10463 & 1.57 \\
\hline $\mathrm{T}_{3}$ - Imazethapyr 10\% SL $75 \mathrm{~g}^{\mathrm{a} . \mathrm{i}} \mathrm{ha}^{-1}$ (EPOE) & 11.65 & 3.41 & 49.02 & 635 & 2217 & 22.25 & 12472 & 1.66 \\
\hline $\begin{array}{l}\mathrm{T}_{4} \text { - Imazethapyr 10\% SL } 50 \mathrm{~g} \text { a.i ha } \mathrm{ha}^{-1}+\text { Adjuvant @ } 2.0 \mathrm{ml} \\
\text { litre }^{-1} \text { of water (EPOE) }\end{array}$ & 11.13 & 3.22 & 48.86 & 618 & 2175 & 22.15 & 11876 & 1.64 \\
\hline $\begin{array}{l}\mathrm{T}_{5} \text { - Imazethapyr } 10 \% \text { SL } 62.5 \mathrm{~g} \text { a.i ha }{ }^{-1}+\text { Adjuvant @ } 2.0 \\
\text { ml litre }{ }^{-1} \text { of water (EPOE) }\end{array}$ & 12.99 & 3.45 & 50.46 & 664 & 2342 & 22.36 & 13865 & 1.74 \\
\hline $\begin{array}{l}\mathrm{T}_{6} \text { - Imazethapyr 10\% SL } 75 \mathrm{~g} \text { a.i ha } \mathrm{ha}^{-1}+\text { Adjuvant @ } 2.0 \mathrm{ml} \\
\text { litre }^{-1} \text { of water (EPOE) }\end{array}$ & 15.06 & 4.08 & 51.14 & 783 & 2528 & 23.92 & 19090 & 2.00 \\
\hline T 7 - Quizalofop-ethyl 5\% EC @ 1000 ml ha ${ }^{-1}$ (EPOE) & 11.33 & 3.12 & 48.72 & 596 & 2110 & 22.15 & 10554 & 1.56 \\
\hline $\mathrm{T}_{8}-$ Standard check (2 IC + $2 \mathrm{HW}$ at 20 and 40 DAS $)$ & 15.43 & 4.23 & 51.18 & 811 & 2638 & 23.71 & 16520 & 1.71 \\
\hline$T_{9}$ - Weedy check & 9.09 & 2.35 & 48.66 & 485 & 1817 & 21.12 & 7038 & 1.41 \\
\hline $\mathrm{T}_{10}-$ Weed free & 15.49 & 4.32 & 51.78 & 833 & 2685 & 23.83 & 13952 & 1.52 \\
\hline S.Em \pm & 0.72 & 0.21 & 1.26 & 39.72 & 113.78 & 1.69 & 1650 & 0.08 \\
\hline $\mathrm{CD}(5 \%)$ & 2.15 & 0.63 & NS & 118 & 338 & NS & 4904 & 0.26 \\
\hline
\end{tabular}

Note: EPOE- Early post-emergence (23 DAS), IC - Inter-cultivation (20 \& 40 DAS), HW- Hand weeding (20 \&40 DAS), DAS- Days after sowing 
Effect of imazethapyr as early post emergence on weed dynamics

Among the herbicide treated plots application of Imazethapyr @ $75 \mathrm{~g} \mathrm{a.i} \mathrm{ha}^{-1}$ + adjuvant @ $2.0 \mathrm{ml} \mathrm{litre}{ }^{-1}$ of water was recorded lower total number of weeds, broad leaved weeds, and sedge at all the growth stages of crop and was on par with standard check (2IC $+2 \mathrm{HW}$ at 20 and 40 DAS). Quizalofop-ethyl @ 1000 $\mathrm{ml} \mathrm{ha}{ }^{-1}$ recorded significantly lower number of grassy weeds at all stages of crop growth which on par with Imazethapyr @ $75 \mathrm{~g}$ a.i $\mathrm{ha}^{-1}+$ adjuvant @ $2.0 \mathrm{ml}$ litre $\mathrm{e}^{-1}$ and standard check (2IC + 2HW at 20 and 40 DAS) (Table $1)$. These results are in conformity with the findings of Venkatesha et al., (2008). The standard check $(2 \mathrm{IC}+2 \mathrm{HW}$ at 20 and 40 DAS) has recorded lesser dry weight of weeds, higher WCE and lower weed index. Among the herbicide treatments application of Imazethapyr @ $75 \mathrm{~g} \mathrm{a.i} \mathrm{ha}{ }^{-1}+$ Adjuvant @ $2.0 \mathrm{ml}$ litre $^{-1}$ of water has recorded lesser dry weight of weeds, higher WCE and lower weed index compared to all other herbicidal treatments (Table 2). These results are in conformity with Venkatesha et al., (2008) and Devi et al., (2012). The higher total weed population, weed dry weight, weed index and lower weed control efficiency was recorded in weedy check (Table 1 and 2). Goud et al., (2013) and Lhungdim et al., (2013) quoted similar findings.

Effect of Imazethapyr as early post emergence on yield and yield attributes

All the herbicide treatments produced significantly higher grain and haulm yields compared to weedy check (Table 3). Weed free check recorded significantly higher grain and haulm yields $\left(833 \mathrm{~kg} \mathrm{ha}^{-1}\right.$ and $2685 \mathrm{~kg} \mathrm{ha}^{-}$ $\left.{ }^{1}\right)$, but on par with standard check (2IC + $2 \mathrm{HW}$ at 20 and 40 DAS) (811 kg ha ${ }^{-1}$ and $2638 \mathrm{~kg} \mathrm{ha}^{-1}$ ) and Imazethapyr @ $75 \mathrm{~g}$ a.i. ha 1+adjuvant@2.0 ml litre ${ }^{-1}$ of water $(783 \mathrm{~kg}$ $\mathrm{ha}^{-1}$ and $2528 \mathrm{~kg} \mathrm{ha}^{-1}$ ). Similarly weed free check recorded significantly higher number of pods and grain weight (15.49 plant $^{-1}$ and 4.32 $\mathrm{g}$ plant ${ }^{-1}$ ), which was on par with standard check (2IC + 2HW at 20 and 40 DAS) (15.43 plant $^{-1}$ and $\left.4.23 \mathrm{~g} \mathrm{plant}^{-1}\right)$ and Imazethapyr @ $75 \mathrm{~g}$ a.i. ha ${ }^{-1}+$ adjuvant @ $2.0 \mathrm{ml}$ litre $^{-1}$ of water (15.06 plant $^{-1}$ and 4.08 g plant $^{-1}$ ) (Table $3)$. This may be attributed to excellent control of broad leaved weeds, sedge and grassy weeds at critical stage of crop growth. These results were in conformity with Venkatesha $e t$ al., (2008), Devi et al., (2012) and Ram et al., (2013). There was no significant difference in thousand grain weight (Table 3). Significantly lower grain yield and yield attributes were recorded in weedy check. This may be attributed to severe crop weed competition stress right from crop establishment to the end of critical period of crop growth.

\section{Effect of Imazethapyr as early post emergence on economics}

Economics of various weed control treatments indicated that significantly higher gross return (Rs 40,707 ha ${ }^{-1}$ ) was recorded with weed free check but on par with standard check (2IC + 2HW at 20 and 40 DAS) (Rs 39,661 ha ${ }^{-1}$ ) and Imazethapyr@75 g a.i.ha ${ }^{-1}+$ adjuvant @2.0 $\mathrm{ml}$ litre ${ }^{-1}$ of water (Rs 38,269 ha ${ }^{-1}$ ). Net return was significantly higher in Imazethapyr @ 75 g a.i. ha ${ }^{-1}+$ adjuvant @ $2.0 \mathrm{ml} \mathrm{litre}^{-1}$ of water (Rs 19,090 ha ${ }^{-1}$ ) but on par with standard check (2IC $+2 \mathrm{HW}$ at 20 and $40 \mathrm{DAS})$ (Rs $16,520 \mathrm{ha}^{-1}$ ) (Table 3). Significant differences were observed in $\mathrm{B}$ : $\mathrm{C}$ ratio due to different weed control treatments. Imazethapyr @ $75 \mathrm{~g}$ a.i. ha ${ }^{-1}+$ adjuvant @ $2.0 \mathrm{ml}$ litre $^{-1}$ of water recorded significantly higher B:C ratio (2.00) compared to all other treatments and was followed by Imazethapyr @ $62.5 \mathrm{~g}$ a.i. $\mathrm{ha}^{-1}+$ adjuvant@2.0 ml litre ${ }^{-1}$ of water (1.74) (Table 3). The higher net return in Imazethapyr@75 g a.i.ha ${ }^{-1}+$ adjuvant @ 2.0 $\mathrm{ml}$ litre $^{-1}$ of water could be attributed to 
higher grain yield and lower cost of cultivation and higher gross return. The variations in $\mathrm{B}$ : $\mathrm{C}$ ratio could be attributed to cost of cultivation and gross return. On the contrary, the lower net return and $\mathrm{B}: \mathrm{C}$ ratio were recorded in weedy check (1.41) due to lower gross return which in turn due to significantly the lower grain yield of greengram. Similar finding was reported by Venkatesha et al., (2008).

Based on results of the field experimentation, it seems quite logical to conclude that profitable, potential and effective weed control in greengram can be achieved by application of Imazethapyr @ 75 g a.i. ha ${ }^{-1}+$ adjuvant@2 ml litre ${ }^{-1}$ of water, another alternative is standard check $(2 \mathrm{IC}+2 \mathrm{HW}$ at

\section{References}

Anonymous, 2013. Area, production and yield of principal crops, Directorate of Economics and Statistics, Department of Agriculture and Cooperation report, New Delhi, 2013, available on the www.agricoop.nic.in.

Devi, K. G., Karunakar, A. P. and Gopinath, K. A. 2012. Integrated weed management in rainfed soybean (Glycine $\max$ L. Merrill). Indian
Journal of Dryland Agricultural Research. \& Development, 27 (2): 5154.

Goud V. V., Murade, N. B., Kharke, M. S. and Patil, A. N. 2013. Efficacy of Imazethapyr and Quizalofop-ethyl herbicides on growth and yield of chickpea. An International Journal of life Science, 8 (3): 1015-1018.

Lhungdim, J., Singh, Y., Kumar, P. and Chongtham, S. K. 2013. Integrated weed management of lamb's Quarters (Chenopodium album) and nut sedge in lentil. Indian Journal of Weed Science, 45 (3): 192-197.

Ram, H., Singh, G., Aggarwal, N., Buttar, G. S. and Singh, O. 2013. Standardization of rate and time of application of Imazethapyr weedicide in soybean. Indian Journal of Plant Protection, 41: 33-37.

Singh, A. N., Singh, S. and Bhan, V. M. 1996. Crop-weed competition in summer greengram (Vigna radiata L.). Indian Journal of Agronomy, 41: 616-619.

Venkatesha M. M., Babalad, H. B., Patil, V. C., Patil, B. N. and Hebsur, N. S. 2008. Bio-efficacy and phytotoxicity evaluation of Imazethapyr in soybean. Indian Journal of Weed Science, 40 (34): 214-216.

\section{How to cite this article:}

Girdhari Lal, S.M. Hiremath and Bheiru Singh. 2018. Effect of Imazethapyr as Early PostEmergence Herbicide on Weed Dynamics and Yield of Greengram (Vigna radiata L.). Int.J.Curr.Microbiol.App.Sci. 7(10): 2518-2524. doi: https://doi.org/10.20546/ijcmas.2018.710.292 